\title{
Examining reference frame interaction in spatial memory using a distribution analysis
}

\author{
Whitney N. Street ${ }^{1} \cdot$ Ranxiao Frances Wang ${ }^{1}$
}

Published online: 2 June 2015

(C) Psychonomic Society, Inc. 2015

\begin{abstract}
Previous research showed competition among reference frames in spatial attention and language. The present studies developed a new distribution analysis to examine reference frame interactions in spatial memory. Participants viewed virtual arrays of colored pegs and were instructed to remember them either from their own perspective or from the perspective aligned with the rectangular floor. Then they made judgments of relative directions from their respective encoding orientation. Those taking the floor-axis perspective showed systematic bias in the signed errors toward their egocentric perspective, while those taking their own perspective showed no systematic bias, both for random and symmetrical object arrays. The bias toward the egocentric perspective was observed when learning a real symmetric regular object array with strong environmental cues for the aligned axis. These results indicate automatic processing of the self reference while taking the floor-axis perspective but not vice versa, and suggest that research on spatial memory needs to consider the implications of competition effects in reference frame use.
\end{abstract}

Keywords Spatial memory $\cdot$ Reference frame $\cdot$ Judgment of relative direction $\cdot$ Systematic bias $\cdot$ Error distribution

Electronic supplementary material The online version of this article (doi:10.3758/s13423-015-0871-y) contains supplementary material, which is available to authorized users.

Ranxiao Frances Wang

francesw@cyrus.psych.illinois.edu

1 Department of Psychology, University of Illinois at UrbanaChampaign, 603 E. Daniel St., Champaign, IL 61820, USA
Spatial locations are inherently relative. That is, a location only exists in relation to a reference frame, typically consisting of a reference point and a reference direction. Reference direction selection has generally been the focus of spatial memory research (Easton \& Sholl, 1995; Klatzky, Loomis, Beall, Chance, \& Golledge, 1998; McNamara, Rump, \& Werner, 2003; Mou \& McNamara, 2002; Presson \& Montello, 1994; Rieser, 1989; Wang, 2007, 2012; Wang \& Spelke, 2000, 2002). Reference directions are usually divided into two main classes, egocentric and allocentric. An egocentric reference is one based on the observer, while allocentric reference corresponds to any external direction.

A commonly used task to test reference direction in spatial memory is the judgment of relative direction (JRD) task. Typically participants memorize a scene of objects from a particular perspectives. They are then asked to take an imagined heading, such as "Imagine standing at A facing B," and respond to a target, "Point to C." The JRD paradigm generally assumes that imagined headings corresponding to an encoded reference direction should have lower error and faster response times, since they can be retrieved directly from memory. Imagined headings that are not encoded in memory must be mentally transformed, which increases absolute error and response time. Therefore, by testing a variety of different imagined headings, JRD performance has been used to identify the encoded orientation.

Using this approach, previous studies suggested that egocentric direction is the default reference without strong environmental cues. For example, Shelton and McNamara (2001; Wang, 2007) showed participants objects inside a circular chamber from one perspective, then asked them to perform JRD from multiple imagined headings. Their judgments were most accurate when the imagined heading was the experienced perspective, suggesting that spatial memory is encoded from the egocentric viewpoint. 
An egocentric reference direction does not always seem to be encoded in memory, however. Mou and McNamara (2002) used a symmetrical object array placed on a rectangular mat inside a rectangular room, with the axes of the object array, mat, and the room all aligned. The participants were instructed to remember the array from the allocentric perspective along the aligned axes, which was disassociated from their egocentric viewpoint. When tested, their performance was best for imagined headings that were parallel or orthogonal to that axis. Furthermore, no performance advantage was found for the experienced, egocentric perspective. These findings were taken as evidence that an object array's intrinsic properties drive reference direction selection with no influence from an egocentric perspective. However, recent studies challenged the performance based interpretations of these perspective taking tasks (May, 2004; Street \& Wang, 2014; Wang, 2005).

Nonetheless, all these studies examined the individual effects of reference directions in spatial memory. However, reference frames do not always exist in isolation and are known to interact within spatial language and attention. For example, when judging whether one object is above another, multiple reference frames can be active and there is a cost in judgments if the primed and response reference mismatch (CarlsonRadvansky \& Jiang, 1998). Moreover, Street and Wang (2014) showed that encoded and transformed representations can interact and produce systematic biases in the JRD tasks. Thus, we hypothesized that egocentric and intrinsic reference may interact in spatial memory as well and may produce a systematic bias in the JRD task. For example, if an egocentric reference is automatically encoded in spatial memory, using an allocentric reference may be possible but there will be an influence of the egocentric reference, leading to biased judgments somewhere in between the two perspectives. The current study developed a new distribution analysis paradigm to test this possibility by examining whether JRD from the encoding perspective is systematically biased toward the alternative perspective.

\section{Experiment 1}

\section{Method}

\section{Participants}

Fifty University of Illinois undergraduates completed the task for course credit. There were 21 males and 29 females.

\section{Stimuli}

The study was conducted using a desktop virtual reality display. The participants sat about $0.5 \mathrm{~m}$ from a 17 -inch monitor and viewed virtual scenes of four colored pegs placed at random locations on a rectangular floor. The viewing perspective was $45^{\circ}$ from the axis of the rectangular floor in horizontal direction, and $30^{\circ}$ above the floor plane in vertical direction (see Fig. 1a). The pegs were red, blue, yellow, and white, the floor was light gray, and the background was cyan.

During the study period, participants could change their perspective within $5^{\circ}$ of the designated perspective, either clockwise or counterclockwise horizontally by moving the mouse to the left or right, or vertically by moving the mouse up and down. This slight variation in viewing angle allowed participants better perception of the depth of the scene through motion cues and allowed them to identify the pegs better in case of occlusions.

The testing display consisted of one stationary dot at the center and one movable dot at the periphery (see Fig. 1c). The color of the dots corresponded to the pegs in the studying display, so that the center dot represented the imagined standing location, while the periphery dot represented the pointing target in the JRD task. The imagined heading was fixed for all trials and was the same direction from which the participants were instructed to memorize the object array. Participants indicated the direction of the target by moving the mouse to the left or to the right, which rotated the periphery dot clockwise or counterclockwise along an imaginary circle around the center dot.

\section{Design}

There were two conditions with 25 participants each. In the Self condition, participants were instructed to remember the scene from their current viewpoint $\left(45^{\circ}\right.$ orientation). In the Floor-axis condition, participants were instructed to remember the display as if they were viewing the array from the intrinsic reference direction aligned with the floor axis $\left(0^{\circ}\right.$ orientation; see Fig. 1a). During testing, they were asked to make relative direction judgments from their respective encoding orientation. For example, in the trial illustrated in Fig. 1a and c, the Self group would imagine themselves standing at the red peg facing the $45^{\circ}$ orientation, while the Flooraxis group would imagine themselves standing at the red peg facing the $0^{\circ}$ orientation. Participants were instructed that the top of the response screen represents their facing direction (like in an overhead map view), and to indicate the direction of the target only and ignore the distance. This type of visual JRD response tasks have been used in previous research (e.g., Street \& Wang, 2014) and showed similar results as those using other response tasks, such as joysticks.

The coordinates of the four pegs were randomly determined for each trial, and the standing-at target and the pointing target were randomly selected from the four pegs in the studying display. 
$\mathbf{a}$

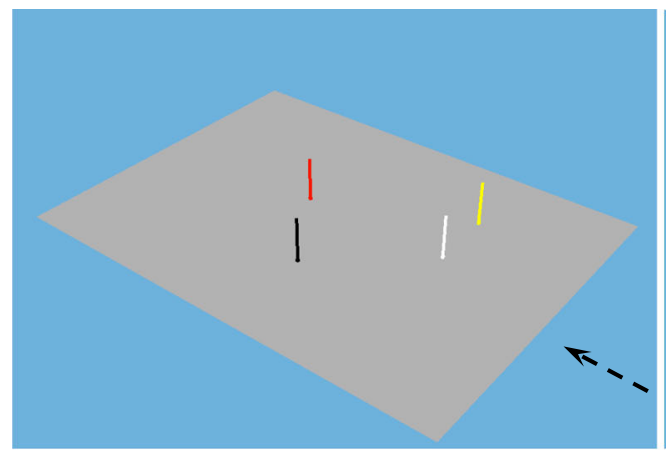

b

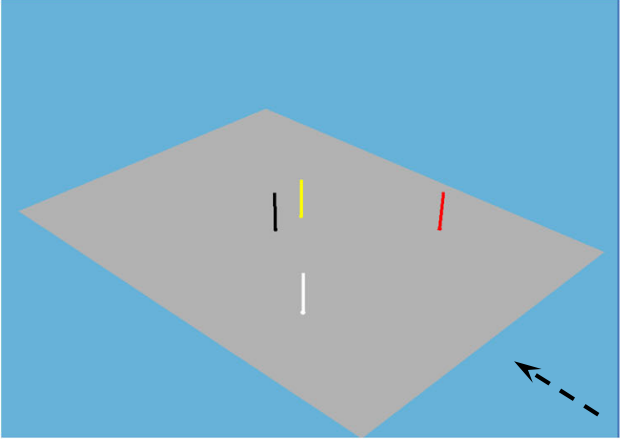

c

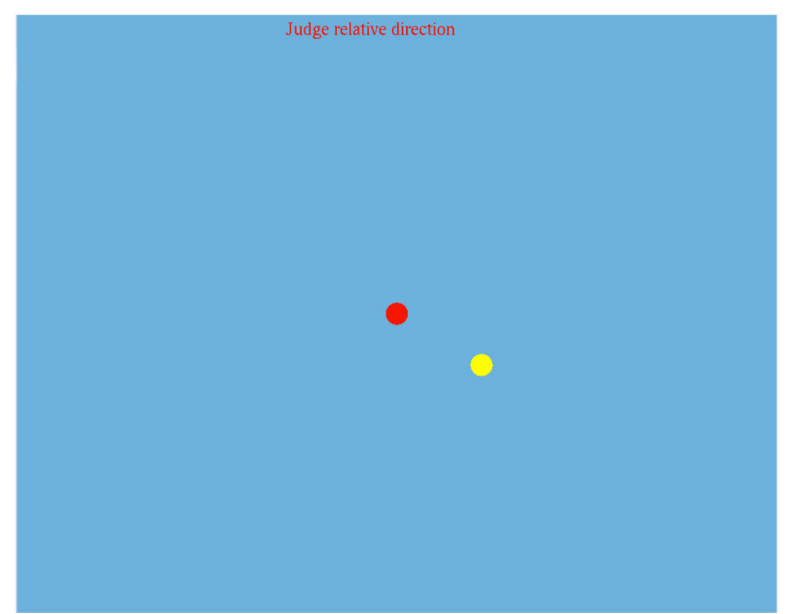

Fig. 1 The sample studying displays for (a) the random peg locations from Experiment 1 and (b) the symmetrical peg positions from Experiment 2. The instructed encoding orientation for the Floor-axis condition is indicated by the dashed arrow. The arrows are for illustration only and were not in the actual stimuli. (c) The testing display for the judgment of relative direction task for both Experiments 1 and 2. Participants were asked to imagine standing at the peg represented by the color of the center dot (red) and indicate the direction of the peg with the color of the periphery dot (yellow) while facing the corresponding encoding direction during the study period

\section{Procedure}

Each trial consisted of a study period of $7 \mathrm{~s}$, a $500 \mathrm{~ms}$ retention period of blank screen, and a test period which lasted until participants made the response. Each studying display was followed by a single JRD test. Immediately after responding the next trial began. Every 20 trials participants could take a self-paced break before continuing through the next block. Participants completed up to nine blocks of twenty trials for a total of 180 trials. If it took them longer than 45 minutes to complete the test, the program was terminated and they were allowed to leave without completing the maximum number of trials.

\section{Data analysis}

For each trial signed error was calculated by subtracting the correct angle from the response angle. Negative error means participants responded further in a counterclockwise direction while positive error means they responded farther clockwise.
If participants were using the instructed reference direction only, responses across all the trials should be centered around the instructed heading with about equal negative and positive errors, therefore there should be no systematic bias overall. In contrast, if participants failed to inhibit the alternative reference direction, their responses should reflect both reference directions and show a systematic bias toward the alternative perspective. More specifically, the Floor-axis group should show a negative bias. For example, as shown in Fig. 1a, the yellow peg should be $150^{\circ}$ from the red peg if participants take the floor-axis perspective. However, the yellow peg is $105^{\circ}$ from the red peg according to the self perspective. Therefore if participants' judgments from the floor-axis orientation are affected by their self perspective, they should show a systematic negative bias. Similarly, the Self group should show a positive bias if the floor-axis exerts an influence in their judgments. Therefore the systematic biases in the spatial judgments can indicate potential interactions between the two reference directions in spatial memory. 
However, there is one alternative explanation based on individual difference. It is known that people differ in their spatial learning and perspective taking abilities (Hegarty \& Waller, 2004; Wolbers \& Hegarty, 2010). For example, if a subset of the participants in the Floor-axis condition failed to follow the instruction due to misunderstanding, inability, or any other reasons, and used their self perspective instead, then their data would cause the group mean to be biased toward the self perspective even if the other successful participants had no bias. This type of systematic bias caused by a small number of participants using the alternative reference does not reflect real interaction between the reference directions, and therefore needs to be ruled out.

To test this alternative explanation, we developed a distribution analysis on signed errors to examine whether individual participants used the instructed perspective. For each participant, signed errors were plotted as a histogram with a kernel curve fit to the data. Kernel fit provides a smooth curve based on the data while making no normality assumptions. Each of these curves was categorized based on the peak of the distribution. If the peak was between $-22.5^{\circ}$ and $22.5^{\circ}$ (i.e., it's closer to the correct reference than to the alternative one), then the participant was classified as successfully using the instructed reference direction. Two independent raters selected which category the peak of the kernel curve fit into. A systematic bias toward the alternative perspective for individuals who followed the instructions correctly would provide evidence against the individual difference explanation and suggest interaction between the reference directions in spatial memory.

\section{Results and discussion}

Participants completed between 124 and 180 trials within the 45-min experimental time limit, with a mean of 176 and 175 trials for the Floor-axis and the Self groups, respectively. ${ }^{1}$ Trials with absolute errors larger than $90^{\circ}$ were considered mistakes and were excluded from the analyses. On average $13 \%$ and $10 \%$ of the trials were excluded for participants in the Flooraxis and the Self conditions, respectively.

In the Floor-axis condition, 17 participants $(68 \%)$ were classified as using the floor-axis perspective. In the Self condition, 23 participants $(92 \%)$ were categorized as using their self perspective. The difference in the proportion of individuals who correctly complied with the instructions was significant $(z=-2.2, p<.05)$, where the floor-axis instruction was followed significantly less often than the self instruction. Both

\footnotetext{
${ }^{1}$ Because all trials were random and independent of each other, it was not critical to equate the number of trials across participants. Therefore, all participants were used in the data analysis.
}

raters independently rated each distribution and agreed on all individual's classifications.

More importantly, one sample $t$ tests of the mean signed errors ${ }^{2}$ using only those participants classified as correctly following instructions for their condition revealed that the Floor-axis condition had a significant negative bias, mean = $-8.9^{\circ} ; t(16)=-10.5, p<.001$, while the Self condition did not, mean $=-0.4^{\circ} ; t(22)=0.34, p=.73$ (see Fig. $2 \mathrm{a}$ and $\mathrm{b}$ ). The bias in the Floor-axis condition suggested that the egocentric reference was processed automatically and competed with the floor-axis reference which participants tried to use to encode and make spatial judgments. In contrast, no bias was found in the self perspective condition, suggesting that the floor axis did not have automatic influence when participants used their egocentric reference.

The systematic bias and poor instruction adherence in the Floor-axis condition may stem from the stimuli themselves. The intrinsic axis of the configuration of the pegs was not strong because the objects themselves were placed randomly on the floor. Therefore even though the floor and instructions provided a cue for the intrinsic reference direction, the array itself might require regularity and symmetry in order for people to form a representation using the intrinsic reference direction and resist the influence of the egocentric reference. Experiment 2 tested this possibility by creating symmetrical displays to see if the bias disappeared.

\section{Experiment 2}

\section{Method}

Fifty different University of Illinois undergraduate students completed the computer task for course credit. There were 25 males and 25 females.

The method was the same as Experiment 1 except for the placement of the pegs on the floor and which objects were possible targets during test. Two peg locations were randomly selected on one side of the floor divided by its main axis. These two positions were then mirrored across this axis (see Fig. 1b). This selection produced displays that were symmetrical and produced an isosceles trapezoid which should simplify the memory task and make the intrinsic axis of the array itself more salient. Finally, this symmetry led to some pairs of objects to be superficially easy and therefore the two objects mirrored along the intrinsic axis were excluded as possible test pairs.

\footnotetext{
${ }^{2}$ Individual participants' mean signed error was calculated according to circular statistics. The group means presented here used ordinary $t$ tests for simplicity. The results were the same when using circular statistics of mean vectors and confidence intervals (Batschelet, 1981).
} 
a

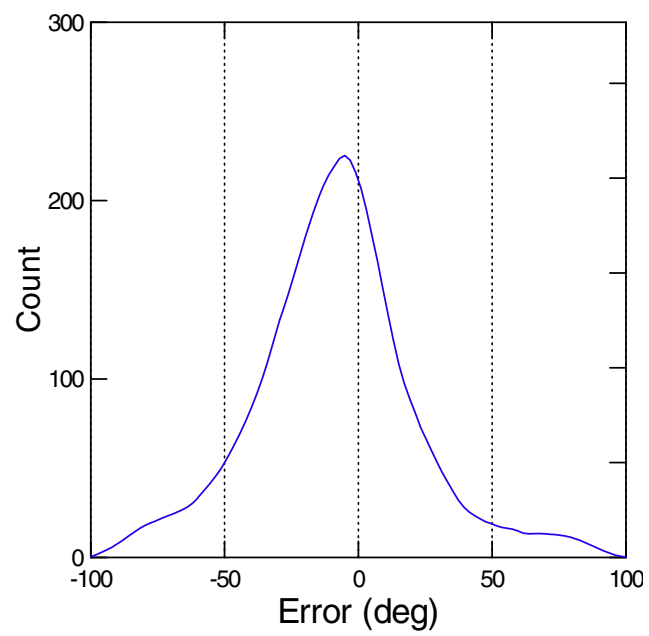

C

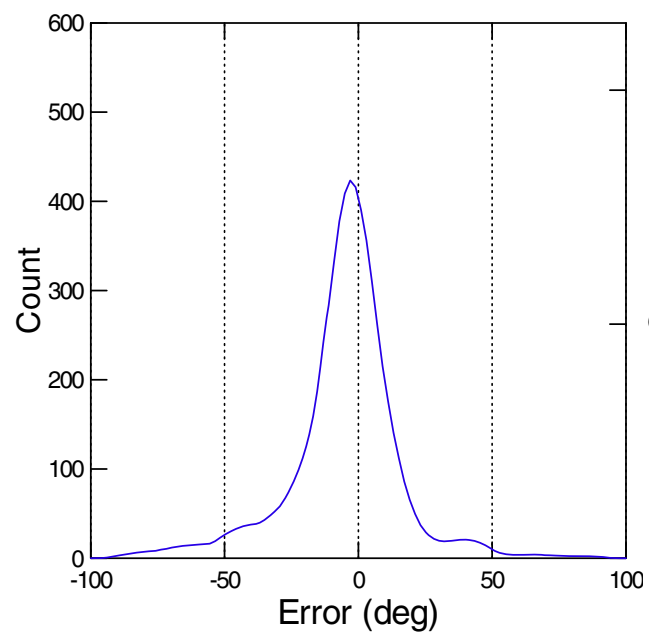

Fig. 2 The kernel curves for all individuals excluding those who did not take the instructed reference direction. These distributions are representative of most participants' individual distributions. The top

\section{Results and discussion}

Participants completed between 155 and 180 trials within the 45 min experimental time limit, with a mean of 176 and 180 trials for the Floor-axis and the Self groups, respectively. Trials with absolute errors larger than $90^{\circ}$ were excluded from the analyses. On average $12 \%$ and $7 \%$ of the trials were excluded for participants in the Floor-axis and the Self conditions, respectively.

In the Floor-axis condition, 16 participants $(64 \%)$ responded correctly according to the intrinsic orientation. In the Self condition, 21 participants $(84 \%)$ responded according to the egocentric perspective. The test for equal proportions of individuals who correctly took the instructed reference across conditions was marginally significant $(z=-1.6, p=.10)$. Both raters independently b

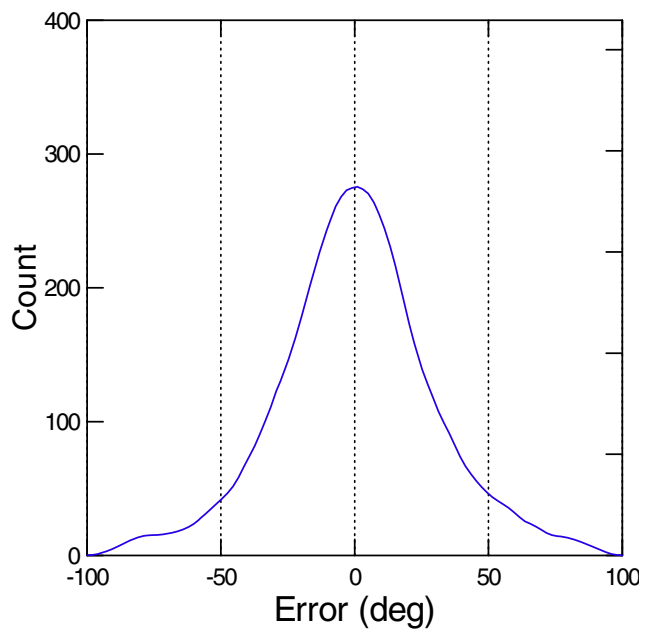

d

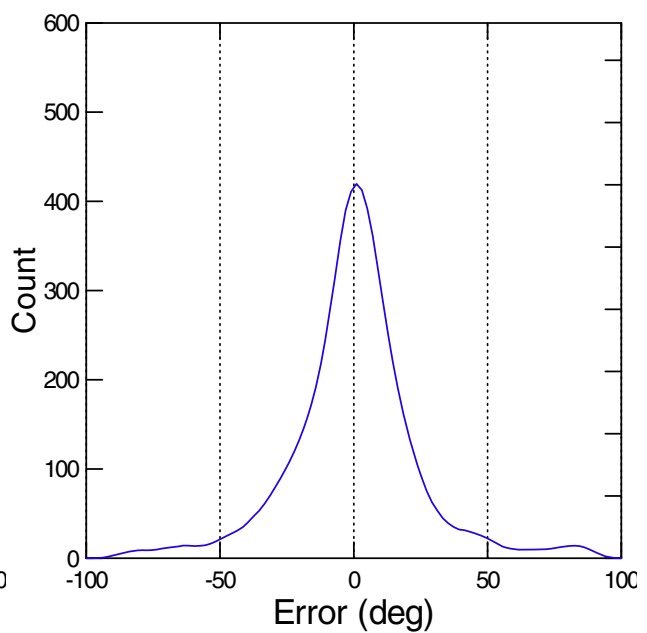

panels are for the random array in Experiment 1 with (a) Floor-axis and (b) Self instructions. The bottom panels are for the symmetrical array with (c) Floor-axis and (d) Self instructions

rated each individual's distribution and fully agreed on each categorization.

More importantly, with only participants who correctly responded with the instructed perspective, the Floor-axis condition still showed significant negative bias towards the self perspective, mean $=-4.9^{\circ} ; t(15)=-6.0, p<.001$, while the Self condition showed no systematic bias, mean $=0.2^{\circ} ; t(20)=$ $0.28, p=0.78$ (see Fig. $2 \mathrm{c}$ and d). Therefore, even with more salient array symmetry the egocentric reference was still automatically processed and affected the use of the intrinsic reference, while the intrinsic reference did not affect the use of the egocentric one.

One potential explanation of the systematic bias in the floor-axis condition is the sensory-motor effect. Sensorymotor effects are typically observed when participants are tested in the studying environment where the relationship 
between their body and the objects are known. Previous research has shown that sensory-motor effects can be eliminated by disconnecting the participants from the object displays, either by testing them in a different room or by disorientation. For example, Mou and McNamara (2002; Street \& Wang, 2014) showed that when the external cues were strong and the participants were tested in a different room, the flooraxis perspective had the lowest absolute error and shortest RT, suggesting there's no sensory-motor alignment cost for the floor-axis perspective in the traditional sense. Therefore Experiment 3 examined the sensory-motor hypothesis under the same experimental condition.

\section{Experiment 3}

\section{Method}

Twenty-five different University of Illinois undergraduate students completed the experiment for course credit. There were 9 males and 16 females.

Participants learned a symmetrical array of 7 real objects on a rectangular mat inside a rectangular room with all three axes aligned. ${ }^{3}$ Then they were brought to a computer room and tested as the Floor-axis condition in Experiment 1, except the following. Each participant completed 160 trials. Instead of two colored dots, two object names were presented on the screen. Participants were instructed to imagine being in the study room standing at the center object, facing the encoding direction aligned with the floor axis, and indicate the direction of the periphery object. The two testing objects were randomly selected among the seven objects for each trial. The data analyses were the same as in Experiments 1 and 2.

\section{Results and discussion}

Trials with absolute errors larger than $90^{\circ}$ were excluded from the analyses. On average $11 \%$ of the trials were excluded. Two raters independently rated each individual's distribution and fully agreed on each categorization. 22 participants ( $88 \%$ ) correctly responded with the floor-axis perspective.

For these 22 participants, there was again a significant negative error, mean $=-1.0^{\circ} ; t(21)=-2.9, p<.01$, suggesting a systematic bias toward the self perspective when trying to use the floor-axis reference, even when learning real object arrays with strong symmetrical and environmental axes, and when participants were tested in a separate environment to eliminate the association between their body sense and the object array. These findings cast doubt on the sensory-motor explanation of

\footnotetext{
${ }^{3}$ The experimental setup and learning procedure were identical to Street and Wang (2014). For details, see Supplementary material.
}

the systematic bias and provide further support that the egocentric reference is automatically processed when people attempt to encode an object array from an external perspective.

\section{General discussion}

Three experiments examined the interaction between reference frames in spatial memory using a novel distribution analysis on signed errors in JRD tasks. Participants were instructed to remember the locations of four virtual objects from either their own egocentric perspective or the intrinsic perspective aligned with the axis of the rectangular floor. When object locations were randomly distributed, more individuals followed the egocentric instruction compared to the intrinsic instruction. Furthermore, participants using the floor-axis orientation showed systematic bias towards the egocentric perspective, while no bias was found in the Self condition. This systematic bias occurred even when learning real object displays with strong intrinsic axes.

These results suggest that the egocentric reference is processed automatically and can exert influence over the use of intrinsic reference but not vice versa. Although most people were capable of using the intrinsic reference when they were instructed to do so, they appeared to have difficulty inhibiting the egocentric reference. As a result, even though their spatial judgments appeared fairly close to the correct responses from the intrinsic orientation, they were nonetheless not exactly centered at the intrinsic orientation and there was a systematic bias toward the egocentric perspective.

There are at least two possible mechanisms such interactions can occur. First, reference directions can compete during encoding, and failure to inhibit the alternative reference will result in a single biased representation in memory. Spatial judgments based on that biased representation are therefore necessarily biased. Second, reference directions may compete during encoding to each form an individual representation. These individual representations are themselves veridical and un-biased. However, when making spatial judgments, both representations may become active and failure to inhibit the alternative representation will result in a combination of the two representations, leading to biases in the responding period (Sampaio \& Wang, 2009, 2012). Further research is needed to dissociate these possibilities.

Although the intrinsic cues in Experiment 3 were very strong, there was still an effect of the egocentric reference on the use of the intrinsic reference. Nevertheless, the size of the bias appeared to decrease when the intrinsic axis was strengthened. Therefore, it is possible that people may use the intrinsic reference exclusively without any influence from the egocentric perspective if the intrinsic cues can be made even stronger. Alternatively, it is also possible that the egocentric reference is always processed automatically, and the relative strength of 
the intrinsic cues primarily affects the weighting between the two references. Whether it is possible to completely eliminate the influence of the egocentric reference requires future studies.

In summary, when encoding and making spatial judgments of a virtual object array from the perspective aligned with the rectangular floor, people showed systematic biases toward their egocentric perspective, both for random and symmetric arrays. However, no bias was found when they used their egocentric perspective. The bias toward the egocentric perspective occurs even when learning real object arrays with strong intrinsic cues. These findings suggest that egocentric and intrinsic reference directions can interact in spatial memory and result in systematic biases in spatial judgments from the encoding perspectives themselves. Research on spatial memory and perspective taking needs to consider these potential interactions to fully capture all references involved.

Acknowledgments Thanks to Aaron Nachsin, Maria Scheet, Lisa Hutcheson, Anthony Lupo, Shea Kaelin, Juliya Kulbachenko, and Jeremiah Pickert for help with data collection. Some of the data were presented at the 54th Annual Meeting of the Psychonomic Society, November 14-17, Toronto, ON, Canada. Comments should be sent to Frances Wang (francesw@cyrus.psych.illinois.edu).

\section{References}

Batschelet, E. (1981). Circular statistics in biology (Vol. 371). London, England: Academic Press.

Carlson-Radvansky, L. A., \& Jiang, Y. (1998). Inhibition accompanies reference-frame selection. Psychological Science, 9(5), 386-391.

Easton, R. D., \& Sholl, M. J. (1995). Object-array structure, frames of reference, and retrieval of spatial knowledge. Journal of Experimental Psychology: Learning, Memory, and Cognition, $21(2), 483$.

Hegarty, M., \& Waller, D. (2004). A dissociation between mental rotation and perspective-taking spatial abilities. Intelligence, 32(2), 175191.
Klatzky, R. L., Loomis, J. M., Beall, A. C., Chance, S. S., \& Golledge, R. G. (1998). Spatial updating of self-position and orientation during real, imagined, and virtual locomotion. Psychological Science, 9(4), 293-298.

May, M. (2004). Imaginal perspective switches in remembered environments: Transformation versus interference accounts. Cognitive Psychology, 48(2), 163-206.

McNamara, T. P., Rump, B., \& Werner, S. (2003). Egocentric and geocentric frames of reference in memory of large-scale space. Psychonomic Bulletin \& Review, 10(3), 589-595.

Mou, W., \& McNamara, T. P. (2002). Intrinsic frames of reference in spatial memory. Journal of Experimental Psychology: Learning, Memory, and Cognition, 28(1), 162.

Presson, C. C., \& Montello, D. R. (1994). Updating after rotational and translational body movements: Coordinate structure of perspective space. Perception, 23, 1447.

Rieser, J. J. (1989). Access to knowledge of spatial structure at novel points of observation. Journal of Experimental Psychology: Learning, Memory, and Cognition, 15(6), 1157.

Sampaio, C., \& Wang, R. F. (2009). Category-based errors and the accessibility of unbiased spatial memories: A retrieval model. Journal of Experimental Psychology: Learning, Memory, and Cognition, 35(5), 1331-1337.

Sampaio, C., \& Wang, R. F. (2012). The locus of the categorical bias in spatial memories. Journal of Cognitive Psychology, 24(7), 781-788.

Shelton, A. L., \& McNamara, T. P. (2001). Systems of spatial reference in human memory. Cognitive Psychology, 43(4), 274-310.

Street, W. N., \& Wang, R. F. (2014). Differentiating spatial memory from spatial transformations. Journal of Experimental Psychology: Learning, Memory, and Cognition, 40(2), 602-608.

Wang, R. F. (2005). Beyond imagination: Perspective change problems revisited. Psicológica, 26(1), 25-38.

Wang, R. F. (2007). Spatial processing and view-dependent representations. In F. Mast \& L. Jancke (Eds.), Spatial processing in navigation, imagery, and perception (pp. 49-65). New York, NY: Springer Science + Business Media.

Wang, R. F. (2012). Theories of spatial representations and reference frames: What can configuration errors tell us? Psychonomic Bulletin \& Review, 19(4), 575-587.

Wang, R. F., \& Spelke, E. S. (2000). Updating egocentric representations in human navigation. Cognition, 77(3), 215-250.

Wang, R. F., \& Spelke, E. S. (2002). Human spatial representation: Insights from animals. Trends in Cognitive Sciences, 6, 376-382.

Wolbers, T., \& Hegarty, M. (2010). What determines our navigational abilities? Trends in Cognitive Sciences, 14(3), 138-146. 